\title{
EDITORIAL
}

\section{ABORDAJES SINTÉTICOS DE INVESTIGACIÓN E INTEGRACIÓN EN ENFERMERÍA}

\author{
SYNTHETICAL APPROACHES IN NURSING RESEARCH \\ AND INTEGRATION \\ Dra. Lorena Chaparro Díaz \\ Directora Unidad de InVESTigación y EXTEnsión. \\ Facultad de Enfermería. Universidad Nacional de Colombia.
}

\begin{abstract}
T a investigación en enfermería en los últimos 20 años en la búsqueda de dismi$\_$nuir la brecha con la práctica profesional viene abordando sus fenómenos de indagación con algunas "nuevas” tendencias de integración como son los estudios meta-análisis, meta-síntesis y todos los que se relacionan con la práctica basada en evidencia.
\end{abstract}

La Etapa de integración busca mantener el dialogo entre la disciplina y sus fenómenos, desarrollar programas educativos alrededor de estos fenómenos, ampliar la perspectiva de la teoría con su visión desde la práctica, un desarrollo acelerado del conocimiento en la práctica, compromiso de los miembros de la disciplina con ese desarrollo y lo más importante que es dar el adecuado reconocimiento a las bases teóricas y filosóficas del cuidado de enfermería (1).

En América Latina y el Caribe vienen llegando hace algunos años estas tendencias que impregnan la formación a nivel de pre y posgrado; se vienen haciendo esfuerzos por modificar el paradigma positivista que por años ha sido el único posible de abordar, para hablar de fenómenos de enfermería basados en necesidades de cuidado. No es lo mismo hablar del cuidado de enfermería en medicina interna a hablar del cuidado de enfermería en situaciones crónicas de salud, o del cuidado crítico a hablar del cuidado de enfermería en situaciones críticas de salud. Para algunos quizás es lo mismo, pero cambia la forma de abordar la persona bajo un paradigma que reconoce la reciprocidad y la existencia de una relación interpersonal donde "el otro me interesa", es decir que considera válido el reconocimiento del otro como un ser humano capaz de construir su propio cuidado ${ }^{1}$. En este sentido,

\footnotetext{
${ }^{1}$ Facultad de Enfermería, Universidad Nacional de Colombia. Programa educativo institucional- programa de enfermería. Bogotá, 2014.
} 
ver de forma cualitativa al ser humano empieza a tomar una connotación importante para la disciplina de enfermería y tiene sentido para la investigación.

La investigación cualitativa, vista como una forma válida para abordar los fenómenos de enfermería y de salud, retoma su fuerza en este momento con metodologías como la síntesis de investigación cualitativa (Meta-síntesis, Meta-etnografía, Meta-sumarios, Meta-método, Meta-estudio, Meta-teoría), tendencias para aumentar el "valor de la utilización” y el "poder" de los resultados de investigación cualitativa. El objetivo central de estas metodologías de síntesis es la revisión de un tópico, agregación, integración, comparación interpretativa y crítica. Existen dos niveles de integración: el de agregación que responde a una síntesis basada en los resúmenes; y la interpretación que es más que la suma de partes, es la transformación de la interpretación de los resultados de estudios primarios (2).

La búsqueda de nuevo conocimiento a partir del que se ha generado de investigación cualitativa debe, por último, responder al nivel de conocimiento en el fenómeno a abordar. No es posible hacer estudios sintetizadores si no hay la suficiente producción de investigación en el fenómeno a indagar, es por eso que usar una metodología sintetizadora requiere de una masa crítica preocupada, como son los grupos de investigación, cuerpos académicos, áreas de trabajo, redes de investigadores, entre otros.

La experiencia en la construcción y desarrollo de estos proyectos lleva a concluir que existen muchos retos para América Latina en la construcción de estudios sintetizadores, como: a) aumentar la producción de investigación cualitativa , aunque hay quienes se han atrevido a traducir estudios primarios en otros idiomas que resultan ser un esfuerzo costoso y dispendioso; b) generar indicadores empíricos que se pongan a prueba con investigación de alto rigor; c) pensar en la evidencia como algo cercano a la práctica, cada nueva forma de ejercer una práctica de cuidado es susceptible de ponerse a prueba; y d) conformar equipos de investigación que combinen enfermeras de la práctica con las de docencia e investigación.

La última versión del Coloquio Panamericano de Investigación en Enfermería en 2012, permitió la socialización de cinco estudios de síntesis cualitativa y uno de síntesis cuantitativa, lo que refleja el gran esfuerzo de América Latina, el Caribe e Iberoamérica por sintetizar el conocimiento de fenómenos de interés para la disciplina, pero reflejan también las deficiencias en producción y formación en estos niveles de investigación.

\section{REFERENCIAS}

1. Meleis A. Theoretical Nursing: Development and Progress. 4a ed. Philadelphia: J.B. Lippincott and Wilkins; 2007.

2. Sandelowski M, Barroso J. Handbook for Synthesizing Qualitative Research. New York: Springer Publishing Company; 2007. 